\title{
Taste learning and memory: a window on the study of brain aging
}

\section{Fernando Gámiz and Milagros Gallo*}

Department of Psychobiology, Centre for Biomedical Research, Institute of Neuroscience, University of Granada, Granada, Spain

\section{Edited by: \\ Edmund Rolls, University of Oxford, UK}

\section{Reviewed by:}

Takashi Yamamoto, Osaka University Graduate School of Dentistry, Japan Raúl G. Paredes, National University of Mexico, Mexico

\section{*Correspondence:}

Milagros Gallo, Department of Psychobiology, Centre for Biomedical Research, Institute of Neuroscience, University of Granada, Avda. del Conocimiento, s/n. P.T. de la Salud, 18100-Armilla, Granada, Spain. e-mail:mgallo@ugr.es
Taste aversion learning exhibits advantages for research on memory brain systems and its reorganization throughout life. A review of the effects of aging on taste memory abilities offers a complex picture showing preserved, impaired, and enhanced functions. Some of the age-related changes in taste memory seem to be associated with an altered temporal processing. Longer taste-illness delays can be introduced for acquisition of conditioned taste aversions and the modulation of taste learning by the temporal context is absent in naïve old rats. It is suggested that an altered hippocampal function is involved in the peculiar performance of these rats. Evidence is also presented which suggests that hippocampaldependent taste memory can be reactivated by previous learning experiences in old rats. Results obtained after reversible inactivation of the dorsal Hippocampus by tetrodotoxin (TTX) in old rats support such a view. Therefore, the interaction between the previous experience and acute brain interventions should be taken into account when studying the effect of aging on taste memory.

Keywords: aging, context, hippocampus, learning, memory, rat, taste, time-of-day
Aging offers a privileged opportunity to study the reorganization of brain memory systems throughout life. At advanced ages the learning and memory abilities have been shaped by several decades of previous learning experiences and face new adaptive challenges due to the modifications of biological conditions. In rodent studies the usual age groups range from young (3-6 months), middleaged (14-18 months), and aged (24-27 months). Even though the effect of age in learning and memory is progressive and agerelated changes in performance have been described as early as at 8-months of age (Gallo et al., 1997) the most pronounced changes occur in middle-aged and aged rats. Thus, 18- to 22month-old animals are often defined as aged even though it has been claimed that they should be considered late middle-aged (Coleman et al., 2004). Contrary to the idea of a global memory decay, normal aging seems to selectively deteriorate some memory functions whilst others remain relatively unimpaired or even enhanced. This is the case in taste aversion learning. Aged rats readily acquire strong, long-lasting aversions to the taste of ingested food leading to visceral distress. The behavioral procedure to induce conditioned taste aversions (CTA) in the laboratory typically involves applying an illness-inducing i.p. injection of lithium chloride ( $\mathrm{LiCl}$ ) after ingestion of a flavored solution. The aversive memory formed after association of taste cues conditioned stimulus (CS) with the aversive visceral signals unconditioned stimulus (US) prevents later ingestion of poisons thus playing a critical role for survival.

Conditioned taste aversions in the rat relies on a brain circuit including areas located from the lower brain stem (nucleus of the solitary tract, parabrachial area) to the higher (amygdala, insular cortex) brain levels. The brain circuit of CTA is described elsewhere (Bermudez-Rattoni, 2004; Lundy and Norgren, 2004; see in this issue Scott, 2011; Yamamoto and Ueji, 2011). CTA also exhibits hippocampal-dependent complex learning phenomena that are selectively impaired by aging. It can be envisaged that the relationship between the hippocampal system and the CTA basic circuit might have been modified throughout life. Thus, the peculiar memory performance of healthy subjects at advanced ages reflects the altered organization of the neural systems involved. A widely accepted view to explain such reorganization is based on compensatory changes to the selective decay of the hippocampal function. Surprisingly, little attention has been paid to the effect of the accumulation of previous learning and memory experiences throughout a long life. Given the plasticity of the brain memory systems, it can be envisaged that changes of the brain systems connectivity have been the obvious outcome of previous learning episodes in order to enhance adaptation to the environmental conditions. Thus, the temporal parameters of learning experiences might become increasingly important throughout life.

This review focuses on the potential time processing changes for understanding the peculiar features of CTA at advanced ages. Special emphasis is given to the effect of previous learning experiences.

\section{TASTE PROCESSING AND NORMAL AGING}

A systematic approach to explore potential explanations of the superior CTA ability related with normal aging should take into account potential modifications of taste processing induced by life events at several steps.

Firstly, aging could alter sensory processing, thus modifying the salience of the taste stimuli to be used in the learning procedures. However no taste sensitivity changes have been reported in aged rodents that could significantly affect the outcome of conventional CTA protocols. Accordingly neuro-physiological responses to various tastes, such as $\mathrm{KCl}$, sucrose, quinine-hydrochloride, $\mathrm{HCl}$, 
monosodium glutamate, and glutamic acid do not change with age in animals (Osada et al., 2003). Even though a decreased olfactory sensitivity has been associated with aging, old rats have been reported to discriminate between odors as readily as younger adult rats (Brushfield et al., 2008) and flavors conventionally used in flavor preference tasks, such as grape and cherry (Renteria et al., 2008).

Secondly, aging could alter the unlearned neophobic response to non-familiar tastes. Taste neophobia is evidenced in decreased consumption of novel taste solutions compared with later exposures as long as the taste becomes familiar. It is well known that stronger aversions are acquired to novel taste solutions rather than to familiar solutions, a well known phenomenon called latent inhibition (Lubow and Moore, 1959). Thus, age-related increases in taste neophobia could account for the higher CTA abilities in old rats.

However, research on this issue has yielded controversial results regarding the impact of aging on taste neophobia. Whilst some studies have found enhanced neophobic responses in aged rats (Collier et al., 2004), no effect of aging has been reported in the amount drunk of a novel taste when using either a grape juice solution (Gallagher and Burwell, 1989; Koh et al., 2009), 0.1\% sodium saccharin (Moron and Gallo, 2007), 1\% sodium chloride (Manrique et al., 2009), 0.5\% sodium chloride, or 3\% cider vinegar solutions (Moron et al., 2002a).

Moreover, the potential impact of aging on taste neophobia and attenuation of the neophobic response might be confounded. This is due to the fact that the full demonstration of the neophobic response to a novel taste requires taking into account not only decreased consumption during the first encounter but also later increases upon subsequent exposures. In fact, a lower rate of neophobia attenuation has been reported in old rats (Pelleymounter and Cullen, 1993) although no age-related differences have been found using a low $\mathrm{NaCl}$ concentration (Manrique et al., 2009).

Regarding the effect of previous experience, early studies showed the relevance of previous aversive taste learning on neophobia to later encountered taste solutions in adult rats (Domjan, 1975; Best and Batson, 1977; Kristal et al., 1980; Franchina and Dyer, 1985). Moreover, there are data supporting an even higher impact of previous aversive experiences on taste neophobia during aging. Thus, previous exposure without consequences leading to habituation of the neophobic response to a sodium saccharin solution disrupted subsequent neophobia to a $\mathrm{NaCl}$ solution both in young and old adult rats. However, a previous saccharin-lithium chloride $(\mathrm{LiCl})$ pairing induced in aged rats a larger increase on later neophobic responses to the salty solution than in young adult rats (Moron and Gallo, 2007). Given that the strength of the previous aversive experience was equated across the age groups, the results could be attributed to a greater impact of aversive memories at advanced ages. Different explanations could account for it being the most suitable a superior learning ability to develop stronger taste aversions at advanced ages. Whatever the explanation, studying the effect of previous taste experiences on the aged rat's willingness to accept novel tastes may contribute to the understanding of controversial results.

To sum up, whilst no aged-related changes in sensory processing seem to be responsible for the superior ability of aged rats to acquire CTA, previous aversive learning experiences could induce increased taste neophobic reactions and/or decreased rates of neophobia attenuation. This might contribute to the formation of stronger learned aversions. This widely unexplored issue is especially relevant because even though many aging studies use naïve animals, it is not unusual to apply previous aversive learning tasks either for dissociating pathological and normal aging or to follow the recommendations for reusing the subjects.

\section{TASTE AVERSION LEARNING AND MEMORY IN AGED RATS}

As mentioned above the acquisition of learned taste aversions seems to be facilitated at advanced ages. As has been reviewed elsewhere (Manrique et al., 2007), stronger aversions are evident in old rats in comparison with young-adult rats during the first extinction test, provided that floor effects are avoided. Although the possibility that this enhancement arises because of impaired extinction cannot be discarded (see below), there are other features of CTA acquisition that point to a superior ability for associating taste and visceral distress in aged rats than in young adult rats. One of the most intriguing features of taste aversion facilitation in aged rats is the possibility of introducing longer intervals between the taste and the $\mathrm{LiCl}$ injections than in younger adult rats. Using a relatively low dose of $\mathrm{LiCl}(1 \%$ b.w., $0.15 \mathrm{M})$ and a $24 \mathrm{~h}$ twobottle test, saccharin aversions have been found in aged rats but not in young-adult rats with taste- $\mathrm{LiCl}$ intervals ranging from 180 (Misanin and Hinderliter, 1989) to $360 \mathrm{~min}$ (Misanin et al., 2002b). This ability to associate a taste with an illness over long intervals develops gradually as rats get older. Thus, rats older than 18 months exhibit taste aversion at the 180 -min interval, whilst only 24- and 30-month-old rats acquire learned taste aversions at 360-min delays (Misanin et al., 2002b).

Different explanations for the age-related facilitation of longtrace taste illness associations have been proposed. Previous results suggest that they cannot be attributed to age differences either in taste sensitivity or increased efficacy of LiCl injection (Misanin and Hinderliter, 1994). Other explanations based on deficits of learned irrelevance (Misanin and Hinderliter, 1995), age differences in the use of interval context cues (Hinderliter and Misanin, 1995b), context-illness associations (Hinderliter and Misanin, 1995a), relative taste novelty (Hinderliter and Misanin, 1993), or memory for specific taste attributes (Misanin et al., 1997) have also been ruled out.

Misanin et al. (2002b) have proposed a longer availability of the taste memory trace in aged rats, because increasing the illness intensity extends the interval over which trace conditioning is evident in old but not in young-adult rats. In order to explain how a memory trace can be available to old rats at a time when it is not longer available to young adult rats, the authors have proposed the slowing down of a metabolic pacemaker. The hypothesized pacemaker is compared to a countdown timer that regulates trace decay after taste processing. The timer would stop at a given duration. Thus, aging can slow the pace at which the clock counts down, thus extending the memory trace decay delay. The effect of aging on this metabolic pacemaker would be independent to that of other circadian clocks or brief interval timers (Misanin et al., 2002b,c). Support for the metabolic pacemaker has been obtained from studies with adult rats showing correlations between decreased metabolic 
rate and the ability to establish long-trace taste aversions. Firstly, at low body temperatures rats displayed learned taste aversions with delays of up to $225 \mathrm{~min}$, which was attributed to a cold-induced slowing of the biochemical clock (Misanin et al., 2002a). Secondly, increasing the metabolic rate by chronic water deprivation reduced the interval that can be introduced in a taste aversion learning protocol (Anderson et al., 2006). Explanation based on an altered sense of time may be related with other reports in animals (Walton, 2010) and humans (Fitzgibbons and Gordon-Salant, 2004; Gooch et al., 2010) pointing to age-related differences in temporal processing using other tasks. No attempts have been made to explore the potential role of age-related anatomical changes in different brain areas in the facilitation of CTA acquisition at advanced ages.

A similar explanation could account for the higher resistance to extinction of learned taste aversions in old rather than in youngadult animals even if no significant differences in acquisition are detected. Thus, Ingram and Peacock (1980) reported that old rats showed delayed extinction of a $\mathrm{LiCl}$-induced saccharin aversion monitored over a period of 32 days. Similarly, resistance to the extinction of a saccharin aversion induced by a low dose of $\mathrm{LiCl}$ has been reported in old rats (Moron and Gallo, 2007). These results contrast with the impaired retention that has been reported in old rats using other learning tasks, such as fear conditioning (Kaczorowski et al., 2011), passive avoidance and learned helplessness (Martinez and Rigter, 1983), among others (Bevilaqua et al., 2008). Given the proposals considering the relevance of a time-induced context differentiation process during extinction, it is conceivable that an altered sense of time could also contribute to slower extinction during aging. An alternative explanation of the slower extinction rate found in older subjects can be related with the greater robustness of the aversion. Nevertheless, even though the age-related superiority in taste aversion learning might rest on the associative mechanisms acting during the acquisition session, enhanced taste memory abilities cannot be excluded given the long intervals supported at advanced ages.

Thus, whatever the explanation, a neural reorganization of the taste memory systems favoring the acquisition and retention of taste aversions at advanced ages seems to be evident. In addition a potential role of changes in temporal processing induced by such reorganization merits attention.

\section{HIPPOCAMPAL FUNCTION AND TASTE MEMORY DURING AGING}

While the hippocampus does not seem to be necessary for acquisition of basic CTA using conventional protocols, the effect of hippocampal damage is evident in adult rats with modified protocols. Firstly, both dorsal and ventral hippocampal neurotoxic lesions have been reported to selectively impair taste aversion learning when 3-h intervals were introduced between taste and illness (Koh et al., 2009). Secondly, temporary inactivation of the dorsal hippocampus by muscimol infusions during acquisition has been shown to enhance learned aversions in a procedure that involved no delay, two different taste solutions, and two conditioning trials (Stone et al., 2005). The authors pointed out to the potential relevance of avoiding ceiling effects due to the relative complex two-taste protocol used. Thirdly, permanent and reversible hippocampal inactivation selectively interferes with a variety of taste complex learning phenomena depending on previous experience (Gallo et al., 1999) as well as on temporal context cues (Molero et al., 2005). Both taste memory enhancement and impairment after hippocampal damage might reflect the interaction between multiple memory systems working in parallel that might induce competitive interference between them. Thus, the hippocampal functions supporting long-delay CTA and complex learning phenomena could be interfering with the acquisition of learned taste aversions (Schoenbaum and Stalnaker, 2005).

It is conceivable that the aging process might modify the potential interaction between the hippocampus and the basic taste memory system. Whilst the evidence from permanent lesion studies does not support an explanation based on hippocampal damage of the age-related changes in taste learning abilities (Manrique et al., 2007), a contribution of an altered functioning of the aged hippocampus cannot be excluded. If this were the case, acute hippocampal inactivation in the behavior of adult animals could be a better model than permanent lesions to study the potential hippocampal involvement in the age-induced facilitation of taste aversion learning (Stone et al., 2005).

\section{PREVIOUS LEARNING EXPERIENCES AND THE TEMPORAL CONTEXT MODULATION OF CTA IN AGED RATS}

Previous results in our lab have shown that a time-of-day shift between taste pre-exposure and conditioning interferes either with learned taste aversions retrieval (Moron et al., 2002b) or with the latent inhibition effect (Manrique et al., 2004). This depends on the extent of the previous habituation to water deprivation procedure (Figure 1). Thus, the comparison between the groups receiving the taste-illness pairings at the same (SAME) and at a different (DIFF) time-of-day than pre-exposure and testing yields an opposite pattern of results in a short-habituation (2 days) versus a longhabituation ( 5 days) protocol. In the former, DIFF groups exhibit weaker aversions than SAME groups whilst in the latter DIFF groups show stronger taste aversions than SAME groups. These patterns reflect the temporal context dependency of CTA (Moron et al., 2002b) and latent inhibition (Manrique et al., 2004), respectively. Both of them demonstrate the ability of the time-of-day to act as a context. The hippocampal integrity plays a crucial role in the temporal modulation of latent inhibition. Thus, neurotoxic lesions of the dorsal hippocampus in adult rats selectively disrupt the effect of the temporal context shift in the long-habituation procedure (Molero et al., 2005). Similarly intact aged rats have been reported to exhibit deficits in the long-habituation protocol. No differences between the aversions shown by SAME and DIFF groups were found (Manrique et al., 2009). This finding does not seem to be explained by a disruptive effect of aging on either latent inhibition (Moron et al., 2002a) or the ability to use the time-ofday as a context. In fact, modulation by the time-of-day was evident in hippocampal aged rats. The pattern of results induced in adult rats by the short-habituation protocol appeared in lesioned aged rats subjected to the long-habituation procedure (Manrique et al., 2009).

Additional data have shown that the temporal context modulation seen in the long-habituation protocol absent in old rats can be reinstated by previous learning experience. Previous training 


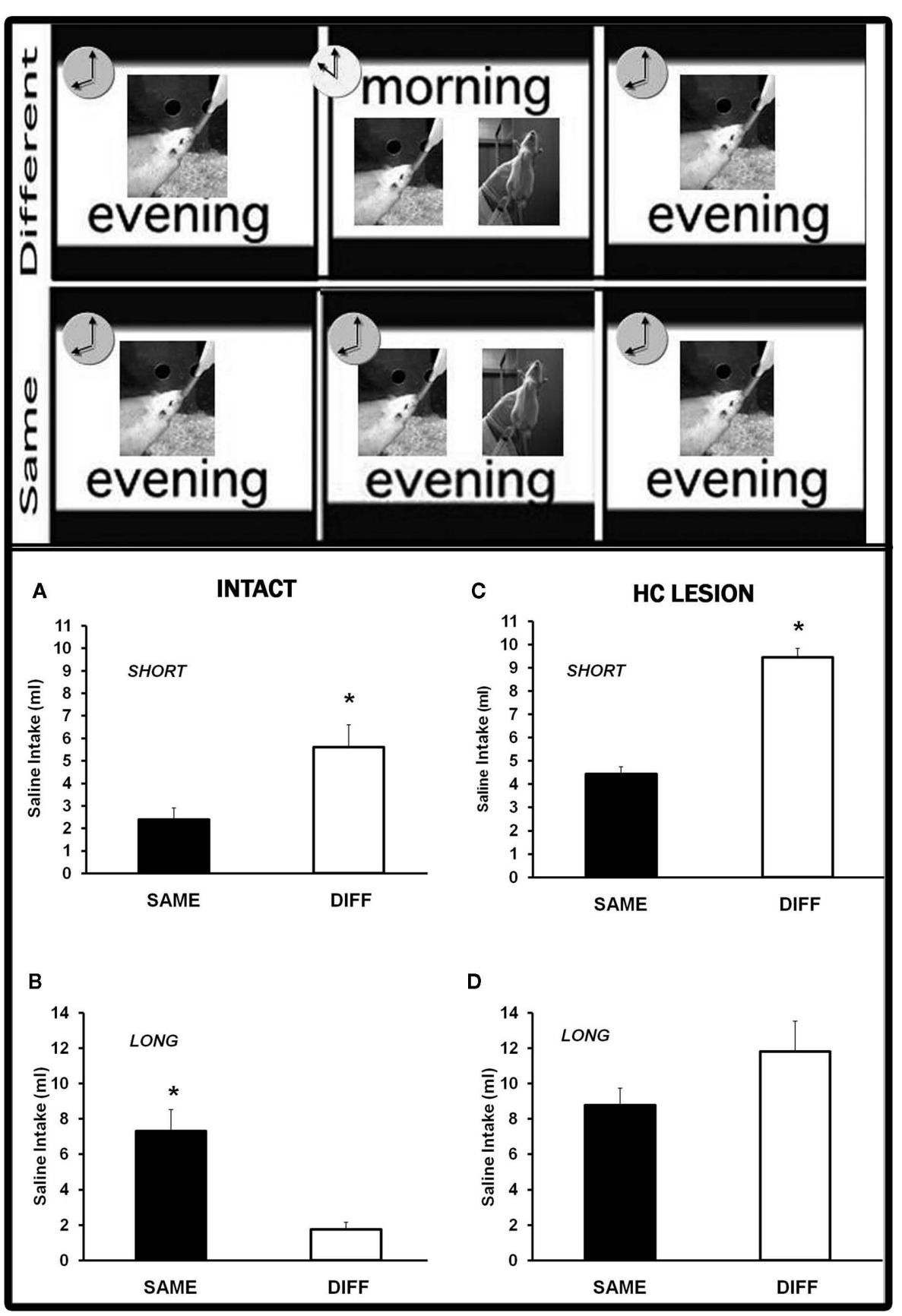

FIGURE 1 |Temporal context-dependent taste learning in adult intact and hippocampal-lesioned rats. Upper panel - the behavioral procedure consisted of four phases: habituation to drink water twice a day, taste solution pre-exposure, taste-LiCl pairing, and testing. In DIFF groups conditioning took place at a different time-of-day to pre-exposure and testing. Groups SAME received all the experimental phases at the same time-of-day. Sessions were performed either at 10 or $20 \mathrm{~h}$. Lower panel - two different protocols were applied depending on the extent of the habituation phase: a short-habituation ( 2 days) or a long-habituation (5 days) protocol. The time-of-day shift induced opposite patterns of results in intact rats subjected either to the short (A) or the long (B) protocol. Hippocampal lesions impaired the effect of a temporal context shift in the long (D) but not the short (C) behavioral protocol. (For further details see Moron et al., 2002b; Manrique et al., 2004, 2009; Molero et al., 2005). in our experiment included several tasks: (a) exposure to a first novel taste solution and subsequent attenuation of taste neophobia, (b) a latent inhibition protocol using a second novel taste solution, and (c) a novel object recognition task. Unexpectedly, trained aged rats exhibited a pattern of differences similar to that seen in adults. The DIFF group showed stronger aversions than the SAME group (Figure 2A). The reinstatement of this adult pattern known to require an intact hippocampus (Molero et al., 


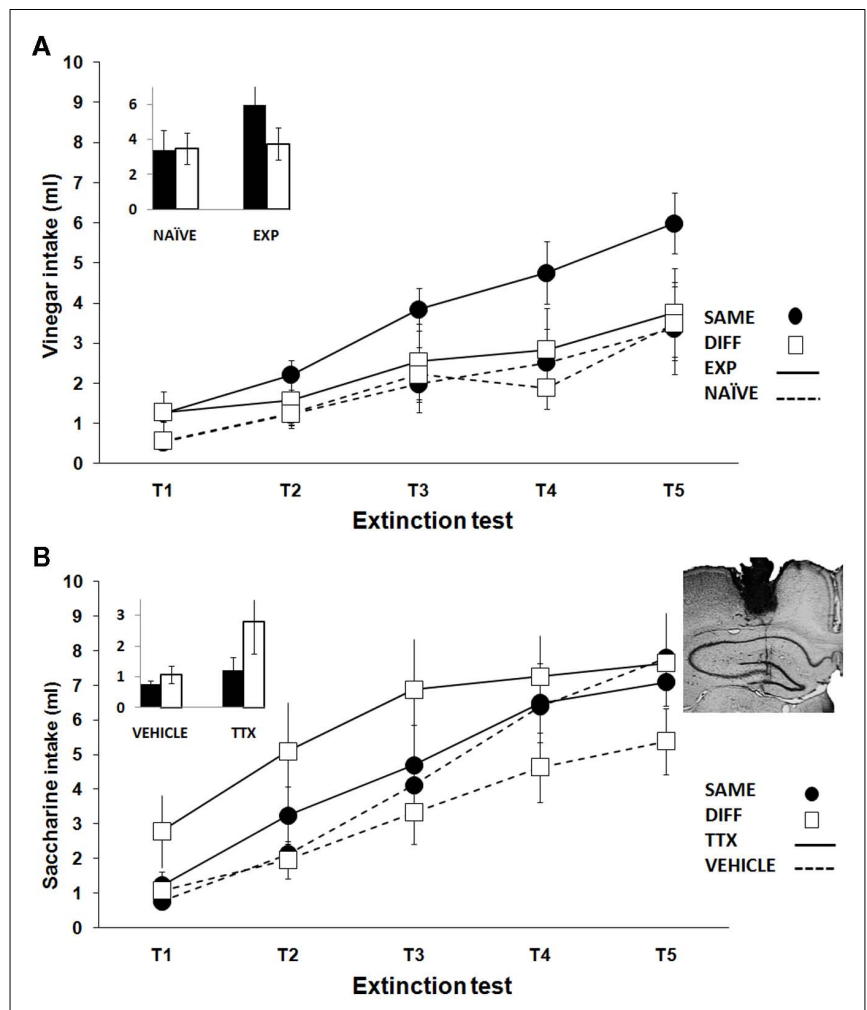

FIGURE 2 | Mean ( \pm SEM) taste solution intake by the aged groups SAME and DIFF using a long-habituation protocol for exploring the hippocampal-dependent time-of-day modulation of taste learning. (A) Reinstatement of the hippocampal adult pattern of results by previous training (EXP groups). Naïve animals (NAÏNE groups) did not exhibit modulation of learning by time-of-day. (B) Emergence of the opposite non-hippocampal pattern of results in trained groups receiving tetrodotoxin (TTX groups) injection in the dorsal hippocampus. Vehicle injected groups (VEHICLE) did not differ between them. The inset highlights the key comparisons on the retention test. A cresyl violet-stained coronal section shows a representative injection needle trace indicating the infusion target area in the dorsal hippocampus.

2005) suggests a potential reactivation of the aged hippocampus function. In our experiments only the above mentioned discrete learning experience was effective. However, a similar 2-monthlong exposure to unspecific environmental enrichment had no effect. Considering that either longer exposure or increased complexity of the enriched environment conditions may be needed, further research is required on this issue.

Moreover, previous learning experience seems to have a critical role in determining the effects of temporary hippocampal

\section{REFERENCES}

Anderson, M. J., Hinderliter, C. F., and Misanin, J. R. (2006). The effects of chronic water deprivation on metabolic rate and longtrace taste-aversion conditioning in rats. Neurobiol. Learn. Mem. 85, 199-205.

Bermudez-Rattoni, F. (2004). Molecular mechanisms of taste-recognition

interventions during aging. Whilst permanent neurotoxic lesions of the dorsal hippocampus in naïve aged rats enhanced the nonhippocampal temporal modulation of taste aversion (Manrique et al., 2009), temporary inactivation by TTX during conditioning induced a similar effect only in trained old rats (Figure 2B). The results cannot be attributed to changes in the attenuation of neophobia during conditioning since hippocampal inactivation during exposure to a $3 \%$ cider vinegar solution had no effect either in the neophobic response or its habituation. It is, therefore, conceivable that reversible temporary inactivation may release functions modulated by the aged hippocampus that were previously reactivated by learning experience. However permanent damage would be required for the reorganization of neural circuits in naïve animals.

The fact that previous discrete learning experiences determine the outcome of hippocampal inactivation in taste learning at advanced ages shows up a complex interaction between parallel memory systems. It is conceivable that the aging process modifies the interaction between hippocampus and the taste memory systems. Therefore, the study of the interaction between the hippocampus and other taste memory systems at advanced ages should take into account the nature of the learning experiences throughout the life.

\section{CONCLUSION}

Temporal processing deficits may be at the root of the peculiar features of older subjects' performance in taste learning tasks. A compromised sense of time in aged animals is supported by both enhanced long-delay taste aversion learning and absence of temporal context modulation.

An altered interaction between the hippocampal system and CTA brain circuits could be responsible for the peculiar temporal attributes relevant for taste memory during aging. Thus, permanent hippocampal lesions facilitate basic non-hippocampal forms of CTA modulation by the time-of-day, thus indicating competition between systems. However transient, hippocampal inactivation produces similar effects only in trained aged rats.

Therefore, memory abilities which have been shaped by several decades of learning experiences throughout life should be taken into account in taste research on aging.

\section{ACKNOWLEDGMENTS}

Supported by the research projects HUM 02763 (Junta de Andalucía. Spain), PSIC2008-03933 (MICINN. Spain), PSI201123702 (MICINN. Spain), and FPU fellowship to Fernando Gámiz (MICINN, Spain). The authors are grateful to Angela L. Tate for her help in editing the English text.

Monteiro, S., Lima, R. H., Medina, J. H., Cammarota, M., and Izquierdo, I. (2008). The role of the entorhinal cortex in extinction: influences of aging. Neural Plast. 2008, 595282.

Brushfield, A. M., Luu, T. T., Callahan, B. D., and Gilbert, P. E. (2008). A comparison of discrimination and reversal learning for olfactory and visual stimuli in aged rats. Behav. Neurosci. 122, 54-62.

Coleman, P., Finch, C., and Joseph, J. (2004). The need for multiple points in aging studies. Neurobiol. Aging 25, 3-4.

Collier, T. J., Greene, J. G., Felten, D. L., Stevens, S. Y., and Collier, K. S. (2004). Reduced cortical noradrenergic neurotransmission is 
associated with increased neophobia and impaired spatial memory in aged rats. Neurobiol. Aging 25, 209-221.

Domjan, M. (1975). The nature of the thirst stimulus: a factor in conditioned taste-aversion behavior. Physiol. Behav. 14, 809-813.

Fitzgibbons, P. J., and Gordon-Salant, S. (2004). Age effects on discrimination of timing in auditory sequences. J. Acoust. Soc. Am. 116, 1126-1134.

Franchina, J. J., and Dyer, A. B. (1985). Aversion conditioning and enhanced neophobia: role of test stimuli. Behav. Neural Biol. 44, 122-131.

Gallagher, M., and Burwell, R. D. (1989). Relationship of age-related decline across several behavioral domains. Neurobiol. Aging 10, 691-708.

Gallo, M., Ballesteros, M. A., Molero, A., and Morón, I. (1999). Taste aversion learning as a tool for the study of hippocampal and non-hippocampal brain memory circuits regulating diet selection. Nutr. Neurosci. 2, 277-302.

Gallo, M., Valouskova, V., and Candido, A. (1997). Fetal hippocampal transplants restore conditioned blocking in rats.with dorsal hippocampal lesions: effect of age. Behav. Brain Res. 88, 67-74.

Gooch, C. M., Wiener, M., Wencil, E. B., and Coslett, H. B. (2010). Interval timing disruptions in subjects with cerebellar lesions. Neuropsychologia 48, 1022-1031.

Hinderliter, C. F., and Misanin, J. R. (1993). Context familiarity and delayed conditioned taste aversion in young-adult and old-age rats. Percept. Mot. Skills 77, 1403-1406.

Hinderliter, C. F., and Misanin, J. R. (1995a). Age-differences and the interstimulus-interval context in long-delay taste-aversion conditioning of rats. Psychol. Rep. 76, 636-638.

Hinderliter, C. F., and Misanin, J. R. (1995b). Age differences and the interstimulus interval context in long-delay taste-aversion conditioning of rats. Psychol. Rep. 76, 636-638.

Ingram, D. K., and Peacock, L. J. (1980). Conditioned taste-aversion as a function of age in mature malerats. Exp. Aging Res. 6, 113-123.
Kaczorowski, C. C., Davis, S. J., and Moyer, J. R. Jr. (2011). Aging redistributes medial prefrontal neuronal excitability and impedes extinction of trace fear conditioning. Neurobiol. Aging. doi: 10.1016/j.neurobiolaging.2011.03.020. [Epub ahead of print].

Koh, M. T., Wheeler, D. S., and Gallagher, M. (2009). Hippocampal lesions interfere with long-trace taste aversion conditioning. Physiol. Behav. 98, 103-107.

Kristal, M. B., Steuer, M. A., Nishita, J. K., and Peters, L. C. (1980). Neophobia and water intake after repeated pairings of novel flavors with toxicosis. Physiol. Behav. 24, 979-982.

Lubow, R. E., and Moore, A. U. (1959). Latent inhibition: the effect of nonreinforced pre-exposure to the conditional stimulus. J. Comp. Physiol. Psychol. 52, 415-419.

Lundy, R. F. Jr., and Norgren, R. (2004). Activity in the hypothalamus, amygdala, and cortex generates bilateral and convergent modulation of pontine gustatory neurons. J. Neurophysiol. 91, 1143-1157.

Manrique, T., Molero, A., Ballesteros, M. A., Moron, I., Gallo, M., and Fenton, A. A. (2004). Time of day-dependent latent inhibition of conditioned taste aversions in rats. Neurobiol. Learn. Mem. 82, 77-80.

Manrique, T., Moron, I., Ballesteros, M. A., Guerrero, R. M., Fenton, A. A., and Gallo, M. (2009). Hippocampus, aging, and segregating memories. Hippocampus 19, 57-65.

Manrique, T., Moron, I., Ballesteros, M. A., Guerrero, R. M., and Gallo, M. (2007). Hippocampus, ageing, and taste memories. Chem. Senses 32, 111-117.

Martinez, J. L. Jr., and Rigter, H. (1983). Assessment of retention capacities in old rats. Behav. Neural Biol. 39, 181-191.

Misanin, J. R., Anderson, M. J., Christianson, J. P., Collins, M. M., Goodhart, M. G., Rushanan, S. G., and Hinderliter, C. F. (2002a). Low body temperature, time dilation, and long-trace conditioned flavor aversion in rats. Neurobiol. Learn. Mem. 78, 167-177.

Misanin, J. R., Collins, M., Rushanan, S., Anderson, M. J., Goodhart, M., and Hinderliter, C. F. (2002b). Aging facilitates long-trace taste-aversion conditioning in rats. Physiol. Behav. 75, 759-764.

Misanin, J. R., Goodhart, M. G., Anderson, M. J., and Hinderliter, C. F. (2002c). The interaction of age and unconditioned stimulus intensity on long-trace conditioned flavor aversion in rats. Dev. Psychobiol. 40, 131-137.

Misanin, J. R., and Hinderliter, C. F. (1989). Role of the CS-US interval in the US preexposure effect. Psychol. Rep. 64, 611-614.

Misanin, J. R., and Hinderliter, C. F. (1994). Efficacy of lithium chloride in the taste-aversion conditioning of young-adult and old-age rats. Psychol. Rep. 75 267-271.

Misanin, J. R., and Hinderliter, C. F. (1995). Lack of age differences in context-illness associations in the long-delay taste-aversion conditioning of rats. Percept. Mot. Skills 80 595-598.

Misanin, J. R., Hoefel, T. D., Riedy, C. A., and Hinderliter, C. F. (1997). Remote and proximal US preexposure and aging effects in taste aversion learning in rats. Physiol. Behav. 61, 221-224.

Molero, A., Moron, I., Angeles Ballesteros, M., Manrique, T., Fenton, A., and Gallo, M. (2005). Hippocampus, temporal context and taste memories. Chem. Senses 30(Suppl. 1) i160-i161.

Moron, I., Ballesteros, M. A., Candido, A., and Gallo, M. (2002a). Taste aversion learning and aging: a comparison with the effect of dorsal hippocampal lesions in rats. Physiol. Res. 51(Suppl. 1), S21-S27.

Moron, I., Manrique, T., Molero, A., Ballesteros, M. A., Gallo, M., and Fenton, A. (2002b). The contextual modulation of conditioned taste aversions by the physical environment and time of day is similar. Learn. Mem. 9, 218-223.

Moron, I., and Gallo, M. (2007). Effect of previous taste experiences on taste neophobia in young-adult and aged rats. Physiol. Behav. 90, 308-317.
Osada, K., Komai, M., Bryant, B. P., Suzuki, H., Tsunoda, K., and Furukawa, Y. (2003). Age related decreases in neural sensitivity to $\mathrm{NaCl}$ in SHR-SP. J. Vet. Med. Sci. 65, 313-317.

Pelleymounter, M. A., and Cullen, M. J. (1993). Effects of idebenone on information processing in aged Long-Evans rats. Pharmacol. Biochem. Behav. 46, 415-421.

Renteria, A. F., Silbaugh, B. C., Tolentino, J. C., and Gilbert, P. E. (2008). Age-related changes in conditioned flavor preference in rats. Behav. Brain Res. 188, 56-61.

Schoenbaum, G., and Stalnaker, T. A. (2005). Thanks for the memories. Learn. Mem. 12, 547-548.

Scott, T. R. (2011). Learning through the taste system. Front. Syst. Neurosci. 5:87. doi: 10.3389/fnsys.2011.00087

Stone, M. E., Grimes, B. S., and Katz, D. B. (2005). Hippocampal inactivation enhances taste learning. Learn. Mem. 12, 579-586.

Walton, J. P. (2010). Timing is everything: temporal processing deficits in the aged auditory brainstem. Hear. Res. 264, 63-69.

Yamamoto, T., and Ueji, K. (2011). Brain mechanisms of flavor learning. Front. Syst. Neurosci. 5, 76.

Conflict of Interest Statement: The authors declare that the research was conducted in the absence of any commercial or financial relationships that could be construed as a potential conflict of interest.

Received: 15 July 2011; paper pending published: 20 August 2011; accepted: 23 October 2011; published online: 08 November 2011.

Citation: Gámiz $F$ and Gallo $M$ (2011) Taste learning and memory: a window on the study of brain aging. Front. Syst. Neurosci. 5:91. doi: 10.3389/fnsys.2011.00091

Copyright (C) 2011 Gámiz and Gallo. This is an open-access article subject to a nonexclusive license between the authors and Frontiers Media SA, which permits use, distribution and reproduction in other forums, provided the original authors and source are credited and other Frontiers conditions are complied with. 